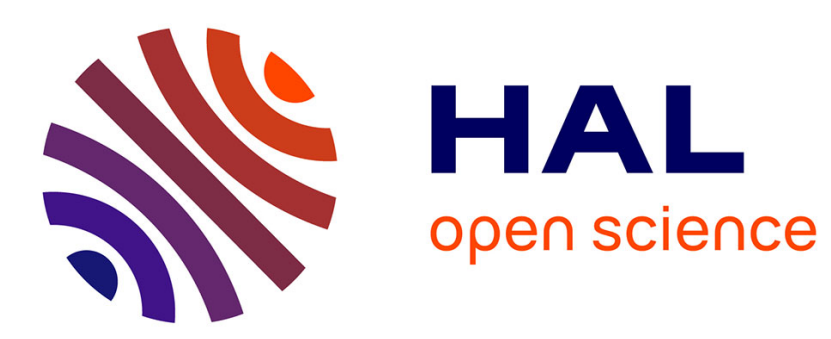

\title{
Exploring conceptual integration in student thinking: evidence from a case study \\ Keith Stephen Taber
}

\section{To cite this version:}

Keith Stephen Taber. Exploring conceptual integration in student thinking: evidence from a case study. International Journal of Science Education, 2008, 30 (14), pp.1915-1943. 10.1080/09500690701589404 . hal-00513359

\section{HAL Id: hal-00513359 \\ https://hal.science/hal-00513359}

Submitted on 1 Sep 2010

HAL is a multi-disciplinary open access archive for the deposit and dissemination of scientific research documents, whether they are published or not. The documents may come from teaching and research institutions in France or abroad, or from public or private research centers.
L'archive ouverte pluridisciplinaire HAL, est destinée au dépôt et à la diffusion de documents scientifiques de niveau recherche, publiés ou non, émanant des établissements d'enseignement et de recherche français ou étrangers, des laboratoires publics ou privés. 


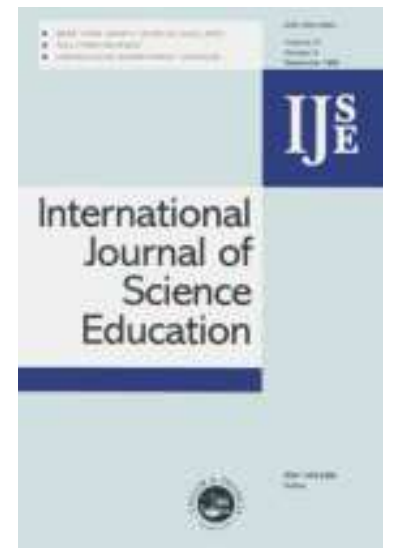

\section{Exploring conceptual integration in student thinking: evidence from a case study}

\begin{tabular}{|r|l|}
\hline Journal: & International Journal of Science Education \\
\hline Manuscript ID: & TSED-2007-0239 \\
\hline Manuscript Type: & Research Paper \\
\hline Keywords: & $\begin{array}{l}\text { qualitative research, chemistry education, physics education, } \\
\text { misconception }\end{array}$ \\
\hline Keywords (user): & $\begin{array}{l}\text { eliciting student thinking, conceptual understanding, conceptual } \\
\text { integration }\end{array}$ \\
\hline
\end{tabular}

\section{今 scholaroNE" \\ Manuscript Central}




\title{
Exploring conceptual integration in student thinking: evidence from a case study
}

\author{
Keith S. Taber ${ }^{1}$
}

\begin{abstract}
:
Two reasons are suggested for studying the degree of conceptual integration in student thinking. The linking of new material to existing knowledge is an important aspect of meaningful learning. It is also argued that conceptual coherence is a characteristic of scientific knowledge and a criterion used in evaluating new theories. Appreciating this 'scientific value' should be one objective when students learn about the nature of science. These considerations imply that students should not only learn individual scientific models and principles, but should be taught to see how they are linked together. The present paper describes the use of an interview protocol designed to explore conceptual integration across two college level subjects (chemistry and physics). The novelty here is that a single interview is used to elicit explanations of a wide range of phenomena. The potential of this approach is demonstrated through an account of one student's scientific thinking, showing both how she applied fundamental ideas widely, and also where conceptual integration was lacking. The value and limitations of using this type of interview as one means for researching conceptual integration in students' thinking are discussed.
\end{abstract}

\section{Key words:}

eliciting student thinking; conceptual understanding; conceptual integration; coherence of scientific knowledge; nature of science; linking chemistry and physics

\footnotetext{
${ }^{1}$ University of Cambridge, Faculty of Education, 184 Hills Road, Cambridge, CB2 8PQ, UK, kst24@cam.ac.uk, http://people.pwf.cam.ac.uk/kst24/
} 
Exploring conceptual integration in student thinking: evidence from a case study

\section{Introduction}

This paper reports on an interview schedule used to explore aspects of students' scientific understanding, and discusses a case study describing aspects of one college student's scientific thinking. The focus of this research is investigating the extent to which students achieve 'conceptual integration' of the science they learn about in school and college.

The paper begins by outlining a case for the centrality of conceptual coherence in science, and so the importance of conceptual integration in science teaching and learning. It is then argued that learners can find integrating their science knowledge difficult, and so further research focused on this area is indicated.

The paper then sets out the basis for the specific study reported here. The design of a research instrument, an interview schedule to investigate student understanding of a range of aspects of chemistry and physics, is explained in terms of previous research findings; and the choice of an in-depth case study approach is justified. The application of the interview schedule and the development of a case study are described.

The case account is then presented to demonstrate the nature of the analysis possible when using this type of interview schedule. The outcomes of the case are discussed in terms of the findings about student application of key scientific ideas and - in particular - evidence of conceptual integration. The potential for developing the approach to investigate progression of learning in terms of conceptual integration is considered. It is concluded that this type of research interview has potential (in certain circumstances) as one approach that can contribute to our developing understanding of aspects of learning in science. 


\section{The importance of conceptual coherence and integration}

This paper explores conceptual integration in learning. There are two distinct reasons for making this a focus of research in science education. These relate to the processes of learning, and to the nature of scientific knowledge.

\section{Conceptual integration and the learning process}

Constructivist models of learning (e.g. Osborne \& Wittrock, 1983, 1985; Pope \& Gilbert, 1983; Smith, diSessa \& Roschelle, 1993) assume that the processes of learning involve the 'building up' of knowledge structures. An individual's knowledge base may be modelled as a network (e.g. diSessa \& Sherin, 1998, cf. Gilbert \& Watts, 1983), so that representations such as concept maps may be used to model aspects of an individual's 'cognitive structure' (Wandersee, 1990).

Constructivist models of learning assume that existing knowledge and understanding is the basis for new learning - or at least what Ausubel (2000) would describe as 'meaningful' learning. Theories of memory processing suggest that long-term retention of knowledge in forms that are readily accessible involves the consolidation of knowledge, through changes (thought to take place during sleep) that increase the levels of integration of recent learning with well-established knowledge structures (Taber, 2003a). From such perspectives we would consider greater integration to support learning, although also acknowledging that inappropriate linkages may well support knowledge recall to the detriment of scientific understanding (Taber, 2004), i.e. leading to alternative conceptions.

From this perspective, teaching that supports learners in seeing how new material links with prior learning should both facilitate new meaningful learning and reinforce the previous learning.

\section{Conceptual coherence and the nature of science}

A second consideration is the nature of scientific knowledge itself. It is considered here that desirable science education will teach students both (a) some science (some of the models and principles that are the products of science and their applications), 
and (b) about science - that is the nature of science (Duschl, 2000). Teaching about the nature of science is recognised as a major goal of science education (Osborne, 2002), although the focus may often be weighted towards teaching about the processes of scientific enquiry (Taber, 2006a). It is considered here that this aspect of science education needs to be supported by an understanding of what may be described as core 'scientific values' (Meichtry, 1998). These might be thought to include objectivity, giving priority to data (over, for example, reputation), sharing of information and so forth.

Another such 'scientific value' concerns the nature of the knowledge being sought as 'scientific'. For example, in recent years there has been considerable attention to the status of the models produced in science (Justi \& Gilbert, 2000). Another aspect of scientific knowledge is the way it is strongly integrated. To be accepted, a new scientific theory would not only be expected to reflect empirical data, but also to demonstrate internal consistency. It is also normally expected to be consistent with existing well-accepted theories. Further, simplicity is often considered to be a criterion for preferring one hypothesis to another (Goodman, 1968), the so-called application of 'Ockham's razor' (Losee, 1993) or 'Occam's razor' (Dunbar. 1995). In science, 'all other things being equal', explaining a wide variety of data with as few basic principles as possible is preferred.

This is not to suggest that inconsistency can never be tolerated: but rather that scientists assume that apparent inconsistencies will either disappear with more thorough analysis, or indicate that further empirical work is needed (Petruccioli, 1993). Looking to simplify (e.g. through the periodic system for the elements) is a key scientific attitude. Although being open-minded would be seen as a scientific value to be encouraged, few professional scientists would readily consider a new theory that was clearly inconsistent with (for example) the principle of conservation of energy.

Thagard (1992) has shown how the notion of explanatory coherence may usefully model development in scientific thinking. This principle assumes that moving towards greater coherence represents scientific progress. Inconsistencies imply 'problem situations' for science (Popper, 1982: 161). Apparent anomalies have been posited as 


\section{Exploring conceptual integration in student thinking: evidence from a case study}

key drivers of major shifts in scientific thinking (Kuhn, 1996), and as problems to be solved within scientific research programmes (Lakatos, 1978).

So coherent, well-integrated, concepts are the norm in the 'public knowledge' of science ("a more or less coherent set of ideas", Ziman, 1968: 2). Research suggests that this is not always the case when we turn to consider students' scientific thinking.

\section{Research into conceptual integration in learning science}

Although there is a considerable body of research into student thinking in science (Driver, Squires, Rushworth \& Wood-Robinson, 1994; Duit, 2006), little of this has looked specifically at conceptual integration. Understandably, much of the research has explored student thinking around particular concept areas. So although much is known about the ways that learners make sense of many science topics, this is largely based on individual studies considering the particular areas of science in isolation.

\section{The research programme}

Although it is argued here that conceptual integration is an important focus for research, it is not considered surprising that it is a relatively ignored area. If research into the learning of science is considered to be a research programme (Lakatos, 1970, 1978) then it would be expected to initially focus on fundamental issues and questions, and then progress over time, with research questions and foci becoming more sophisticated and specific as fundamental areas of theory are slowly developed (Taber, 2006b). So a good deal of existing research and scholarship has been focused around questions such as:

- What ideas do learners' bring to science classes, and what is the nature of these ideas?

- How much commonality is there between learners' ideas in science?

A good deal of empirical work has addressed these areas. Some of this research (i.e. in relation to the second question) has tended to use methods suitable for collecting 
data from large enough samples to discuss how widespread certain ways of thinking are among learners. For example, an alternative framework based around specific ideas about force and motion has been reported to reflect the thinking of most school age learners (Watts \& Zylbersztajn, 1981).

Researchers are now increasingly looking at more difficult questions concerning how teaching interacts with existing knowledge and exploring the progression of learning over extended periods of time. These types of foci tend to require more sophisticated qualitative approaches, often being based on in-depth study of particular teaching and learning contexts (Duit, Roth, Komorek \& Wilbers, 1998) or exploring the nuances of thinking of individual learners (Petri \& Niedderer, 1998; Harrison \& Treagust, 2000; Taber, 2001a).

In a similar way, targeting research on conceptual integration also requires more demanding approaches than suffice for finding out about student thinking relating to one topic. This strand of research, like that investigating progression in learning, requires more detailed investigations with individual learners. As a minimum, students needs to be asked in some depth about two potentially related areas of science, or probed in depth about a science focus where understanding inherently depends upon knowledge of other topics.

The research reported here is part of a project (ECLIPSE, Exploring Conceptual Learning, Integration and Progression in Science Education) that has conceptual integration as one of its key concerns (Taber, 2005a). One of the approaches being explored in the project is discussed below: the development of interviews that can survey understanding of a range of potentially linked science topics.

\section{The need to explore how students integrate their scientific knowledge}

One concern with research into learners' ideas in science (alternative conceptions and so forth) has been the extent to which these ideas are used consistently. For example, Engel Clough and Driver (1991) looked at the degree of consistency with which students apply particular conceptual frameworks in different contexts, but within specific topic areas (they looked at the topics of pressure, heat and inheritance). The 
Exploring conceptual integration in student thinking: evidence from a case study

focus in the present study is the coherence across topics that would indicate conceptual integration. Part of the motivation for this concern derived from previous research into student understanding of a specific topic. The focal topic was chemical bonding. From the researcher's perspective, this was a chemistry topic where learners would be expected to draw upon more fundamental physics learning.

However, what was actually found in the Understanding Chemical Bonding research was that students did not tend to bring the relevant physics concepts to mind when learning about the chemistry (Taber, 2003b), which led to the development of alternative conceptions that were inconsistent with the assumed 'pre-requisite' physics learning (Taber, 1998a). Indeed, there was some evidence that, from the student perspective, being expected to draw upon existing physics learning during chemistry was sometimes considered as an additional and unreasonable demand (Taber, 1998b).

So, for example, although students understood the nature of forces between charged particles, they invented alternative mechanisms to explain chemical processes (such as bond formation) and the stability of chemical structures, although these topics were being taught on the basis of physical principles. So students believed that reactions occurred because of the 'needs' of the atoms involved, and octet structures were considered to automatically have inherent stability - even in extreme cases such as a hypothetical $\mathrm{Na}^{7-}$ ion (Taber, 2002a). Even in a context where students did use notions of electrical forces in explanations (ionisation energy) they commonly invoked an alternative electrostatics (the sharing around of nuclear force) to the Coulombic principles of physics. Given these findings, conceptual integration seemed to be a potentially fruitful focus for further research.

\section{Methodology}

Following on from the findings of the previous research, it was decided to develop an interview questionnaire that could be used to explore the extent of conceptual integration in students studying chemistry and physics. It was decided to target this at 'sixth form' level (16-18 year olds) for reasons that are explained below. 


\section{Design of the interview}

The specific findings of the earlier research suggested some useful concept areas to include, but other topics were included to increase the scope of study. The topic areas were chosen from aspects of chemistry and physics, because:

- it was considered important to include topics that students would perceive as most relevant to more than one distinct science discipline;

- it was connections between chemistry and physics that had been highlighted as potentially problematic in the earlier research;

- the researcher's own subject knowledge and teaching background were strongest in these subjects.

A sequence of questions were planned, with follow-ups in case certain points were not addressed in the original answers. The full sequence is presented in the appendix, but the contexts included were

- apple hanging on a tree, and dropping

- a weight suspended from a spring

- $\quad$ a torch (flashlight)

- the solar system

- a balloon 'stuck' to a wall after charging

- a parachute jump

- charging someone with a van der Graaf generator

- chemical reactions: burning of magnesium, hydrogen reacting with fluorine; sodium reacting with chlorine

- stability of structures: sodium chloride crystals; chlorine molecules; iron crystals; sulphur crystals; ice crystals; atoms; nuclei

- 'physical' changes: melting ice, salt dissolving, ionisation

The topics were chosen to offer examples of contexts likely to be familiar from school science, including both 'mechanics' and 'electricity' contexts in physics and including 
consideration of a range of materials with the main types of bonding and structure considered in school and college chemistry.

It was considered that these topics offered potential for considerable conceptual integration through the three fundamental concepts of forces, energy and particles. These are recognised in the English context as providing the basis for secondary level teaching in the physical sciences (DfES, 2002). This is reflected in figure 1 . The figure offers a concept map illustrating how the topics discussed in the interview are linked through these basic scientific ideas.

The map is arranged around the concept of forces, but the main links between 'force' based explanations and 'energy' based explanations are shown. That is, the concepts of energy and force are fundamentally linked so that it would be possible to substitute, or complement, force-based explanations with energy-based explanations (e.g. when forces do work, energy is transferred; forces acting are balanced at local minima of potential energy). In school or college science terms, we might suggest they offer alternative and equally valid 'explanatory stories' (Millar \& Osborne, 1998).

The 'map' is divided into two domains where topics shift from the discussion of macroscopic objects, to the theoretical world of particle models that are used to explain properties in the macroscopic realm. The nature of this transition is wellrecognised to be a source of difficulties for many learners (Taber, 2001b; Harrison \& Treagust, 2002).

(Figure 1 here)

Figure 1: A concept map illustrating themes linking the interview items

The decision to ask about the 'physics contexts' first was deliberate (cf. Tomlinson, 1989), so that respondents would have a chance to use physics ideas in these contexts before being asked about chemical phenomena. Research suggests that learners may hold competing representations of knowledge (multiple frameworks or manifold conceptions) so it would be quite possible for a student to be able to explain the same phenomena in several distinct ways (Taber, 2000a). The student's 'conceptual 
ecology' (Hewson, 1985) might include a range of resources that can potentially be selected from when constructing a response (Hammer, 2004). Presenting the physics contexts could potentially 'prime' the students, and make it more likely they would use these fundamental ideas in chemistry contexts if their personal knowledge structures offered sufficient conceptual integration to support such applications. (The distinction between a student not having access to a potential linkage, and not drawing upon that linkage in offering a particular response is considered further later in the paper.)

(Unlike much interview research into student understanding in science (Bell, 1995), where semi-structured approaches are used (with a limited number of focal questions, and the expectation of considerable follow-up), a much more structured approach was selected (a methodological decision reviewed later in the paper). The intention of the schedule as a research instrument was to provide a structured basis for collecting data about student thinking over a range of topics within a realistic time span.

Research designs involve compromises between different objectives. A less structured approach allows more in-depth exploration of thinking about particular foci, but typically limits the range of an interview. In this general area of research the intention is to explore aspects of a students' knowledge and understanding, yet 'cognitive structure' (White, 1985) is typically complex and multi-facetted. So an interview necessarily provides a 'snapshot', reflecting the interaction of the actual object of research, and the particular probes we used (cf. Phillips, 1987). To understand some of the complexity of student thinking about a topic we often need to revisit in alternative contexts.

Yet there are also clearly pragmatic considerations in this type of research. Interviews are 'gifts' from our informants (Limerick, Burgess-Limerick \& Grace, 1996) and their time is valuable. As researchers we have ethical responsibilities to those who we interrogate (Taber, 2002b) - in terms of both the time and mental exertion we ask from them. In this research, collecting data about thinking over a range of topics was more important than being able to spend time approaching particular topics from a range of perspectives. In negotiating access to potential informants, and in asking the students to volunteer for the research, it was envisaged that the interview process 
should be completed within a one-hour timeframe. However, the schedule was not totally fixed: there was scope to allow further unplanned follow-up questions where these were needed for clarifying responses.

\section{Use of the interview schedule and selection of informants}

The decision to work with 'sixth form' students was based on a number of considerations. Most of the specific previous research that had led to a concern with this issue was undertaken with students studying a science subject at 'A level' (Advanced level, i.e. university entrance level examinations in the UK system) in a College context. These are students who have opted to continue in post-compulsory education; who have selected to continue studying science subjects; and who have been accepted by the college as suitable for study at this level on the basis of success in school leaving examinations.

This present research, then, also concerns students who are working at an advanced level having shown interest in science, and having been successful in academic work in school. These are students where we might expect significant evidence of conceptual integration, and who should cope with the challenge of a broad-based interview of around an hour's duration. In particular, in view of the selection of topics in the schedule, it was decided to look for students who had selected to study both chemistry and physics at college level.

Contact was made with a large Sixth Form College in the City where the researcher is based. The College has several hundred students following courses in sciences at 'A level'. The Head of Physics at the College arranged an invitation to be circulated among students who were completing the first year of the two-year course. This led to four students volunteering for interviews in July 2003. Outline information about the four students is presented in table 1 .

(table 1 here)

Table 1: The student volunteers interviewed using the schedule 
Mutually convenient interview times were arranged individually with the four students. The interviews were held in the University, which was convenient to both parties (as the Education Faculty and the Sixth Form College are neighboring institutions).

The interviews took place in a comfortable private location, and were recorded on audiotape with the informants' knowledge and permission. The interviews began with the explanation of purpose and collection of some personal data, and ended with a 'debrief' about the experience. In between, the interview schedule was followed. The informants were told they could ask to take a break or stop the process at any time (an option not exercised). The interview duration varied from about three quarters of an hour, to well over an hour according to how much information the students offered in response to the questions and whether follow-up questions were considered necessary. All four students reported being comfortable with the process. Notes were made during the interviews, but the recordings were later transcribed to give a more detailed written record.

The four individuals presented distinct and interesting cases. Even within a single interview, the amount of data collected with the schedule was considerable. Although a structured schedule can provide the basis for comparing across a sample, it was considered that the most appropriate approach was to analyse the individual learners as discrete cases - as the main focus was coherence between the responses within an interview. Therefore a case study approach was selected (Simons, 1980; Gomm, Hammersley \& Foster, 2000; Yin, 2003). Case study is used when phenomena are complex (e.g. an individual's cognitive structure) and differences between individual cases are unlikely to be open to analysis in terms of simple readily identifiable factors. Although it is not possible to generalise from a case study, individual cases can offer insights that may be of wider significance (and can be tested in other contexts). For present purposes, the intention of discussing one of the cases in detail is to demonstrate the value of the approach as a means of exploring an important aspect of learning science: rather than suggesting that the specific findings of the single case study are in themselves widely significant. 


\section{Exploring conceptual integration in student thinking: evidence from a case study}

\section{Selection of a case}

The selection of a case in case study research can be problematic - as by definition case study is used when instances are unique as so all cases are potentially illuminating (Taber, 2007). Often in case study research, the case is identified at the outset: being chosen because it has specific features that are expected to illuminate key issues that may have wider significance. In the present research the volunteering students were not known to the researcher in advance, and there was no reason to expect any particular student interview to provide the basis for a more informative case. Indeed it was found that each of the interviews provides data that are illuminating in terms of exploring conceptual integration.

In such a situation, the selection of a case for discussion needs to be based upon some rationale. So, for example, in Petri and Niedderer's (1998) case study of a student's learning pathway they collected data from a group of students over an extended period (with the risk that some students would be absent at key data-collection points or leave the class), and then later made a selection of 'Carl' for their case study. Carl was chosen as a student who made verbal contributions in class, and who was not at either extreme of the attainment range.

The first criterion offered assurance that Carl that would be well represented within the classroom data collected. In the Understanding Chemical Bonding project a case was selected for detailed discussion partly on the basis of unusually rich data set available - including over twenty in-depth interviews (Taber, 2000a; 2001a).

The second criterion offered by Petri and Niedderer implies some form of 'typicality', and so a case potentially offering insights likely to have wider relevance. Case study focuses on the individual, and so (unlike approaches that seek statistical generalisation) does not claim to identify a sample strictly 'representative' of a wider population. Another case from the Understanding Chemical Bonding projects was selected for discussion in the literature, despite featuring an apparently idiosyncratic example of a tenacious alternative conception, because it illustrated how within a single topic area some concepts remained very stable whilst others were labile (Taber, 1995). The decision to avoid extreme cases (as in Petri and Niedderer's study), or to 
actively seek cases where atypical features may be especially illuminating, needs to be made on theoretical ground in particular studies.

In the present research volunteers were invited from a college with an excellent record of examination success at university entrance level. All four volunteers aspired to science related degree courses at University (see Table 1). This approach to sampling was based less on identifying typical students at this level, than recruiting students who might be considered well placed to have developed strong understandings of the science (high achievers, with strong motivation to do well in their science studies).

One advantage of using volunteers (which needs to be balanced against the risk of interviewees being atypical of the wider cohort) was that all four students were able to speak confidently and coherently about the topics included in the interviews. This is not to suggest that they had ready answers to all the questions, or that they were always confident in the correctness of all their responses. However, these students were able to respond clearly to the questions, and to expand upon and explain their answers where this was requested. The interviews generated a rich data set, with quite detailed responses to some questions. Each of the four students offered answers to the full range of questions in the interview protocol, so all provided suitable data for the research.

Each case offers features of interest. The selection of Alice as a case in this present study is based on one of the purposes of the present paper: that of illustrating the potential value of a broad interview protocol such as used in this research. The protocol in the appendix was used as the interview guide in all four interviews. The full set of questions was used in each, with just minimal changes to wording and occasional slight variations in order (to meet the normal expectations of conversation - for example where a question has clearly been fully answered in response to the previous question). However, there was some variation in the extent to which student answers were follow-up by unscripted questions.

In the first interview, such follow-ups were restricted to points where it was felt clarification was needed to appreciate Alice's response. This restriction was partly related to a concern that time or student engagement would be exhausted before 
completing the full set of questions. This concern was found to be unjustified, and in the subsequent interviews, more opportunities were taken to probe student responses, to collect more detailed and nuanced information.

The discussion of Alice's responses, then, offers a case study of conceptual integration across chemistry and physics based on the interview protocol as designed, and therefore offers the best guide to the potential of the protocol when used in a 'pure' form. (The implications of this methodological decision are considered later in the paper.)

\section{Analysis of the case}

Alice (an assumed name) was the first student to be interviewed. She had achieved the top grade in the 'double science' option (a 'broad and balanced' science course) of the school leaving examination, and was studying biology and German, as well as chemistry and physics (see Table 1). The interview took almost exactly one hour. After the interview, Alice described the interview process as 'challenging'.

As Pope and Denicolo (1986) have discussed, the role of the analyst in qualitative work involves balancing the importance of detailed accounts directly drawing upon the words and ideas of the informants, with the need to provide reports which are concise and presented in ways to help the reader. The building of the case study took place in two stages, following a general approach used in the author's previous research (e.g. Taber, 1995).

Firstly the interview transcript was reworked into a narrative account of the interview based around Alice's verbatim responses, but following the chronology of the interview schedule in the order of the questions. This stage of analysis is largely descriptive - similar to the initial 'open coding' stage used in much qualitative analysis (Glaser \& Holton, 2004). The main purpose was to convert the format from a transcript into a narrative form to aid readability. For example, consider the following brief extract, 


\begin{abstract}
Alice described how when a parachutist jumps from a plane, she "starts descending due to gravity... and then, she'll be affected by things like uplift, well, resistance, air resistance....and if she's high up enough, should reach sort of terminal velocity" when "her downward force is equalled by the upward force of air resistance, working against her, so you get a balance." The downward force was "gravity acting on her mass which is weight, her weight". When asked to explain about upthrust, Alice suggested that idea "actually, links in more with water", where there was "a surface area, and then you've got a body of water, beneath, acting on it", and withdrew the suggestion: "scrub that one".
\end{abstract}

The total length of the narrative account was a little over 2300 words. The next stage of the analysis involved reorganising the case material into themes in terms of the main concepts used in Alice's explanations (see the next section). This process produced a case account that was reduced (in this case to about 1000 words), and which summarises the ways Alice used ideas in her interview. The case account is presented in the next section to illustrate the range of information that was elicited in a single interview tightly structured by the schedule presented in the Appendix.

\title{
A case study of conceptual understanding across physics and chemistry topics
}

\section{Forces}

Alice used the language of forces and energy in response to many of the questions posed. Alice considered the role of opposing forces in situations such as an apple hanging from a tree (where gravity balanced the tension in the stalk), or a loaded spring where weight is opposed by tension in the spring. She reported that gravity operated between large bodies such as the sun and planets, and the earth and moon. She identified weight, air resistance and upthrust as forces acting on a parachutist, although she then decided the latter only applied in water.

Alice did not explain recoil of a stretch spring in terms of internal forces, even thought she did refer to atoms in her discussion of elastic and plastic strains. However in other structures she identified intermolecular forces, such as van der Waals' forces and the forces between polar water molecules, and intramolecular forces such as covalent bonding, or electron transfer due to one atom pulling on an electron more 
strongly. Forces were also involved when a solid dissolved. Alice said that the attraction between charges held an atom together, but she could not suggest how protons and neutrons were held together in the nucleus.

\section{Force and motion}

Alice applied ideas about unbalanced forces being a cause of motion, and specifically acceleration, in a number of situations - falling apples, parachutists etc. She described how increasing air resistance on a parachutist would lead to balanced forces and so a terminal velocity. Although Alice identified unbalanced forces with acceleration, she apparently considered orbital motion to be the result of balancing centripetal and centrifugal forces, that kept the orbiting body moving round. Alice described velocity as speed with a direction, and acceleration as a change in speed, and so did not seem to consider a change of direction alone as sufficient criterion for an acceleration.

\section{Interactions between charges}

According to Alice, balloon could be attached to a wall by charging, so that there was a 'glue effect' with an attraction between areas of positive and negative charge. A negatively charged balloon could be attached to a neutral wall because it was relatively positive, although an individual negative proton would not be attracted to a neutron in this way. Static electricity could make someone's hair stand on end as the hairs would have the same charge and be forced away from each other.

Alice recognised attractions between charges at atomic level, for example suggesting that a positive hydrogen ion would attract a negative fluorine species. She described van der Waals' forces in terms of momentary dipoles due to the shifting electron clouds around molecules: again attraction between positives and negatives.

Delocalised electrons in a metal acted as a glue between the positive nuclei. In ice there was a lattice of dipoles that have lined up, positives to negatives. These dipoles could attract and pull away parts of the sodium chloride so that it would dissolve. Atoms were also held together by the attractions between positive and negative charges. Electrons could be attracted away from atoms. The difficulty increased with 
each electron removed as the effective nuclear charge increased the amount of charge acting on each remaining electron - although the shells of electrons could lead to shielding making the situation more complicated.

\section{Energy}

Alice used the notion of stored energy, which could be released as movement, to explain how a stretched spring could recoil. Alice also used the energy concept to explain how a battery could be used to light a torch lamp. In discussing electrical current Alice referred to voltage and e.m.f., and talked of a gradient, or a difference that she thought was similar to the idea of potential energy. Heat was required to allow magnesium to burn in air. Similarly, a spark would provide activation energy to break bonds in a gas mixture. When ice was melted heat provided energy to allow the molecules to escape the forces holding them in place. Random motion was involved when a solid was dissolved. Energy was needed to remove electrons from atoms, and the amount of energy increased on subsequent ionisations.

\section{Particle models}

Although Alice did not refer to forces between atoms in discussing the elastic behaviour of materials, she did refer to "slippage, between atoms" to explain why some materials did not revert to their original form after being strained.

She referred to current as a movement of electrons, and explained the conductivity of a metal in terms of delocalised electrons that could flow. Electrons were also involved in the party trick of sticking a rubbed balloon to the wall, being transferred between balloon and sweater when they were rubbed together.

Alice explained the burning of magnesium simply in terms of it readily oxidising. However, in general, she apparently considered chemical reactions as due to the interactions between electrons in different atoms, molecules or ions. Atoms had a central nucleus and electrons in orbits or orbitals. 
Alice suggested hydrogen was a positive ion in the context of a reaction, although it occurred as a diatomic gas. If activation energy was provided the bonds in the molecules would break giving atoms, but these would be ions as they were lacking in electrons and so did not have full shells. Similarly, she reportedly imagined the reaction between sodium and chlorine in terms of ions interacting - chlorine had negative charges, and a metal was naturally positive.

Alice said that $\mathrm{NaCl}$ comprised of molecules, which in turn comprised of atoms of sodium and chlorine, held together by electron transfer to complete the atom's outer shell - which led to one atom attracting another which had got its electron. The electron transfer depended on the forces pulling on an electron due to the nuclear charge and electronic structure of atoms. On dissolving the molecules would disassociate.

Alice reported that chlorine molecules were held together by covalent bonding, which was where electrons were shared to complete the outer shells atoms. The bonding in a metal was an ionic lattice with delocalised electrons acting as glue between the positive ions. Water molecules were bent, and polar, comprising of positive hydrogen, and negative oxygen that had more electrons.

\section{Discussion of the case}

The case account above demonstrates how the interview schedule (see the Appendix) is suitable for eliciting information about a wide range of phenomena. This technique does indeed offer insights into the degree of conceptual integration, as well as highlighting where Alice had alternative conceptions about the topics. The case account above can be used to highlight points of interest, which can be further illustrated in Alice's own words.

Alice used key ideas of force, energy and particles widely in her explanations (cf. Figure 1), and certainly did use ideas about forces and energy in some of her explanations in chemical contexts. Her particle models of chemical systems seemed to often be at odds with the accepted versions - it is known that this is a very difficult area to master (Lijnse, Licht, de Vos \& Waarlo, 1990; Harrison \& Treagust, 1996, 
2002). She used the term ions for atoms that did not have full shells (cf. Taber, 1995). For example, hydrogen atoms were "ions that we consider as atoms" as "they're lacking an electron. They should [sic] have two electrons to fill the first shell". She also saw $\mathrm{NaCl}$ as molecular, a common alternative conception (Taber, 1997).

Her explanations of chemical reactions seemed to be a mixture of ideas based upon electrical interactions and alternative ideas based upon the significance of full shells. So in explaining the reaction of hydrogen and fluorine - which she apparently thought was an interaction between ions, i.e. atoms without full shells - Alice talked of "hydrogen which is a positive ion, and then fluorine which is highly negative, so the two are going to attract, and then bond together and form hydrogen fluoride".

She described ionic bonding as where "rather than electron being shared between the two component atoms, you've got one being transferred to the other, to complete its outer shell". She also, however, referred to how electrons were transferred "because you've got a stronger - pulling force or attraction in one atom". Previous UK research has suggested that at the end of compulsory schooling many students understand chemical change and bonding in terms of atoms seeking full shells, and that during A level there may be a slow progression to thinking instead in terms of electrical interactions (Taber, 2001a). Although progression cannot be judged from a single interview, it seems Alice held and applied multiple frameworks for thinking about these phenomena, and so it is likely her thinking here was similarly in a process of transition.

Although she discussed electrical interactions between and within atoms and ions, she appeared to hold the common notion (Taber, 1998b) that nuclear charge was shared out among electrons, so on ionisation, 
Alice explained an apple hanging from a tree and an extended spring in terms of the balance of forces, and went on to describe how a parachutist would first accelerate and then "should reach sort of terminal velocity" when "her downward force is equalled by the upward force of air resistance, working against her, so you get a balance". Although Alice seemed to have a reasonable understanding of balanced and unbalanced forces, and applied this idea across a range of contexts, she misapplied the principle in the context of orbital motion.

Here she demonstrated another common alternative conception, considering circular motion as being the result of balanced forces. She thought that the planets were subject to a balance between "centripetal force ... keeping something in an orbit, but also the forces opposing that, which I think is centrifugal... which would send it out of orbit". This seemed to be a misconception of the status of circular motion, rather than any difficulty in anaylsing the situation. In her thinking, orbital motion is not accelerated, and so is ontologically similar to linear motion (cf. McCloskey, Carmazza \& Green, 1980)

Finally, it is worth considering her explanation for why a balloon rubbed on a jumper is able to remain attached to a wall. This is a 'party trick' familiar to most children, but probably not specifically discussed in many science classrooms. Alice recognised this as being an electrostatic effect,

\footnotetext{
"some sort of interaction if you like with the electrons and things, and you have a positive and negative charge which allows, a glue effect if you like, attraction between two areas, one of positive and one of negative."
}

The charging was easily explained as "when you're rubbing the balloon you're transferring electrons either onto it or away from it". To explain why the balloon 
would stick to the wall, Alice proposed that "because you've got opposite charges, you've got the say negatively charged balloon, and then your positively charged wall". Although the wall "hasn't had anything done to it as such", Alice suggested that "maybe in comparison to your very negatively charged balloon, it's still likely to attract." Alice agreed that she was suggesting that "it's relative", that because the neutral object is positive by comparison with the negative object, they're effectively both charged.

There are three aspects of this explanation that are worthy of comment. Firstly, that although the notion of relative charge did not match scientific thinking, such logic would have applied in terms of comparing electrical potentials $(0 \mathrm{~V}$ is negative compared to $+12 \mathrm{~V}$ for example), and it is possible this is an example of (consciously or otherwise) developing an alternative conception by making an analogy with a related area of science (Taber, 2004) - we might tentatively see this as inappropriate conceptual integration.

However, it is also interesting that although Alice suggested this as a feasible explanation in the context of the balloon 'trick', she did not extend the idea to another apparently similar context. Alice did not think that the nucleus of the sodium atom could fall apart, at least "not spontaneously". She could not offer any idea for what holds the nucleus together. She was asked if her earlier idea could apply, as if the neutrons were neutral, then they were more negative than the positive protons: but Alice rejected this idea as "way too simple, and too nice".

As pointed out above, Alice saw $\mathrm{NaCl}$ as a molecular structure, and explained the integrity of the solid in terms of "strong enough ... intermolecular forces holding things together". She suggested that these forces might be "van der Waals' forces" which were where, 
So in the context of intermolecular bonding, Alice discussed how neutral species could be attracted due to induced dipoles. However, she did not consider a possibility along these lines to explain how the charged balloon could somehow have an attraction with a neutral wall. Here the potential linkage was missed.

\section{Alice and her peers}

Alice presents an interesting case of a 'successful' college student of science. Her school-leaving examination record had won her a place as a sixth-form college with a strong academic reputation, to study science subjects. She aspired to enter University to study a science-based subject, and in interview she seemed interested in her studies. In these respects she was very similar to her peers, Benjamin, Charles and Dorothy.

One case of an individual learner does not of itself provide strong grounds for advising teachers how they might modify classroom practice. Although there is not space here to consider the cases of Benjamin, Charles and Dorothy in any depth, it is interesting to note that aspects of Alice's case were reflected in the other interviews, as well as being consistent with findings from earlier research. This will be illustrated by a brief consideration of four of the specific findings in Alice's case:

- balanced forces and orbital motion

- chemical bond formation

- sharing out of nuclear charge

- the balloon party trick

Alice suggested that orbital motion was an example of forces being balanced. It has been well reported that students may believe circular motion to a natural form that does not require a driving force (e.g. McCloskey, et al.1980). Alice did appreciate the 
relationship between force and accelerated motion, but did not see circular motion, when at constant angular speed, as being accelerated.

Neither Dorothy nor Benjamin described orbital motion as the outcome of balanced forces, and Benjamin explicitly described this as an example of accelerated motion. Charles however, like Alice, described orbital motion in terms of balanced forces at work, in spite of not being able to identify the force that might be balancing the gravity. Charles' uncertain explanation reflects another A level Physics student (studying in another college) interviewed as part of the wider ECLIPSE project: Tim suggested a balance between gravitational force and the orbiting body's velocity (Taber, 2005b).

The comments of these students suggest that even when students move beyond common intuitive notions of a force being needed to maintain constant velocity (Watts and Zylbersztajn, 1981), to appreciate the link between force and acceleration, teachers need to be alert to how some students will nevertheless construe orbital motion as due to a balance of forces.

Previous research on chemical bond formation and the nature of bonding strongly suggested both (a) that by school leaving age students commonly attempt to explain bonding and bond formation in terms of atoms 'trying' to fill their shells (Taber, 1998a); and (b) that subsequent teaching about bonding grounded in principles from basic physics leads to a slow transition between offering explanations in terms of the desirability of full shells and the adoption of explanations in terms of electrical interactions (Taber, 2001a). In the present study Alice exemplified this, offering responses drawing upon both acceptable curriculum science (i.e. electrical forces), and notions of atoms forming bonds to complete their shells. Although the interviews with Alice's peers elicited responses that differed in detail, Benjamin, Charles and Dorothy all demonstrated a tendency to explain chemical reactions and bonds using a mixture of acceptable curricular science and notions of atoms wanting full shells. The availability of relevant concepts and principles - Benjamin, for example, referred to both enthalpy and entropy - does not necessarily prevent students using inappropriate ideas, such as atoms striving to fill their electron shells. 
Similarly, all four students presented evidence of thinking about atomic ionisation in terms of the sharing out of nuclear force. This particular topic was highlighted in previous research (Taber, 1998b) as being offering a fertile context for exploration of conceptual integration across physics and chemistry. Alice's responses reflected this earlier research -when discussing atomic ionisation, she demonstrated a nonCoulombic notion of nuclear forces being shared-out between electrons. Each of the four students interviewed with the present schedule described successive ionisations in slightly different terms - but all four implied that in some sense the removal of one electron from an atom or ion would lead to the remaining electrons increasing their share of the nuclear attraction.

In both of these contexts (bonding and ionisation) it seems that students who are able to demonstrate acceptable ways of discussing charge interactions in Coulombic terms when asked about directly observable phenomena (such as hair standing 'on end'), do not readily adopt these same principles when providing explanations in terms of the particle models used so widely in chemistry.

As a final example, the question about the balloon trick seemed to offer a context that all four students found familiar without having a ready explanation. This seems to be a phenomena known from childhood experience that is not discussed within the school science context. Benjamin initially offered an explanation that seemed similar to Alice, that although the wall was neutral, the attraction of the charged balloon could lead to an overall lower 'imbalance' of charge. However as he discussed his ideas, he appeared to have an insight, suddenly suggesting 'Oh it induces the charge in the wall or something.' Charles' response was even closer to Alice's, suggesting that if the balloon was positively charged it "would be attracted to ... anything that is less positively charged than itself". Charles was aware of van der Waals' forces, but his description ("when you've got a sea of electrons which sort of flows around each other") did not explicitly demonstrate an appreciation of induction. Dorothy offered a rather different explanation of the balloon 'trick' when the charged balloon would attract opposite charge from the wall, and in doing so discharge itself. Dorothy did refer to van der Waals' forces as an example of intermolecular forces, but again did not explicitly discuss this as induction. 
So in terms of this particular context, Alice demonstrated knowledge of how electron distribution could be distorted in the context of van der Waals' interactions, which she did not draw upon when asked about the phenomenon of the balloon attached to a neutral wall. Benjamin did make this link, whilst is less clear if Charles and Dorothy had the available conceptual resources to call upon.

As might be expected, the different students offered different patterns of explanation and did not all make (or miss) the same potential links. So for example, Benjamin discussed the extension and recoil of a spring in terms of balanced and unbalanced forces. However he did not seem able to transfer this form of explanation from the macroscopic level to the level of particles in the spring. He was therefore asked about the analogous situation when an elastic band was stretched (to see if he would invoke a molecular level explanation), and in this context he adopted a different principle upon which to base his response: minimisation of energy. Although these two principles offer linked and parallel 'explanatory stories' (Millar \& Osborne, 1998), it is interesting that Benjamin shifted between them when the physical contexts seem so similar.

Even more salient was how in the context of the stretching spring, Benjamin aborted his attempt to draw upon notion of bonding "it means that there's the bonding in, except it's not really bonding because it's all metal isn't it - I can't really explain that one", yet later in the interview he explained how iron crystals were "metallically bonded, and this is all about the lattice of little ions ... and the sea of electrons". This volte-face seems to be something more that not recognising the relevance of available knowledge.

\section{Implications for teaching}

There are some specific points that could be drawn from this study about the teaching of particular topics, although that was not the particular focus of this research. For example, the apparent plausibility of the full-shells explanatory principle has been highlighted in the earlier research, and the present study corroborates earlier findings that able students who have been taught about college level curriculum models (based on electrical interactions) will none-the-less commonly resort to explanations in terms 
of atoms 'wanting' to full their shells. Recommendations have been made for how teachers can approach the teaching of this material to emphasise the accepted models and challenge the common alternative framework (Taber, 2001b).

Perhaps what the present study underlines is the extent to which a students' conceptual map of the science they study is likely to be much less well integrated than the teacher's. Teachers may often assume students will make links that they do not, and may perhaps underestimate the time delay before such linkages are consolidated enough to provide the basis for further learning (Taber, 2004). Although research into conceptual integration is at an early stage, sensible advice to teachers would be to always be very explicit about the way new material builds upon previous study (cf. Ausubel, 2000), and to reinforce these links when developing a topic (rather than assume that because the connection was pointed out at the start of the topic, students will continue to bring it to mind in later lessons).

The type of approach to planning teaching recommended in the secondary curriculum in England, based on identifying key ideas (such as energy, forces, particles) that act as organising themes for teaching and integrating themes for learning (Grevatt, Gilbert \& Newberry, 2007), offers promise as a very useful way of supporting student learning through linking together the science they are taught. A key feature of the nature of science is how we look for models of the world that are built upon a limited number of key and widely applicable principles, so such a teaching approach is if school and college teaching is to better offer an authentic image of science. Ideally teachers should not only be explicit about relevant linkages when they are teaching, but also be explicit about the epistemological significance of highlighting the connections as something we expect and look for in science.

\section{Methodological issues and implications for further research}

Any individual study is likely to be of limited significance when seen in isolation, being better understood as part of a research programme - drawing upon previous research in conceptualisation, and indicating potentially fertile directions for further work (Taber, 2007). Indeed it is increasingly recognised that educational research programmes that can inform practice are likely to draw upon a range of 
methodologies (NRC, 2002). The present study is part of a progressive research programme into learning in science that aims to inform the developing theoretical underpinning to science pedagogy (Taber, 2006b).

The present case study does suggest that interview protocols of the form used here, covering a range of contexts, can be valuable in exploring conceptual integration. The particular interview schedule used in this study (see Appendix) did elicit data that could be interrogated to investigate the extent to which consistent ideas were used across a range of chemistry and physics topics. Whether such an approach could have been used with younger students, or with less confident learners, is a question for further studies, but Alice and her peers seemed to be unfazed by, and indeed reported enjoying the challenge of, the marathon tour of topics.

The findings suggest such approaches make it possible in principle to identify situations where a student does not demonstrate 'target knowledge' because it is not accessed in a particular context rather than there being a lack in the basic conceptual resources. Alice did understand the principle of electrical induction - but did not offer that as a basis for explaining the balloon 'trick'. In that context, this possibility did not seem to come to mind as a basis for an explanation - she did not make the link. Such 'failures to connect' are potentially significant in science teaching and learning.

Having established the value of this type of schedule, a possible future direction for research is to attempt to identify patterns in the way students do or not form such links: patterns that might exist beyond the specifics of individual topic links and might indicate features that aid or impede students making such connections. Knowledge of such patterns could clearly inform effective curriculum planning and teaching practice.

Clearly Alice is one case, and her peers did not demonstrate the same specific patterns of concept integration: as illustrated by Benjamin's responses when asked about a stretched spring. Comparing Alice's responses to those of Benjamin, Charles and Dorothy demonstrates both similarities and differences between students. One possible avenue for further research is to use interviews of this form to 'survey' a wider sample of learners to investigate possible patterns that may be detected. 
Survey methodology is often associated with large-scale approaches using statistical techniques, which are based on rather different ontological and epistemological assumptions than the idiographic approach used in the present study. Clearly the quantity and complexity of data elicited in research interviews of the type discussed here, and the subsequent analysis required, would legislate against large-scale application of the schedules. However, techniques drawn from grounded theory methodology (using 'theoretical sampling' to move towards 'saturation' of findings) provide an alternative iterative approach to developing models of general relevance (Taber, 2000b) that need not be based upon large samples.

Despite the clear value of this approach there are also limitations. In Alice's case it may be conjectured that there is a connection between the way the electron-nuclear interactions were seen as due to one of the charged bodies (the nucleus here, rather than being an interaction between charges), and the reference to the pulling force "in one atom" during electron transfer. This type of potential connection, only noticed during analysis, could have been followed-up if there had been a sequence of interviews, which might also have allowed revisiting the topics through slightly different contexts (to test something of the range of application of Alice's thinking).

A related methodological issue is the extent to which student responses should be followed up where links to basic principles are not initially made. In Alice's interview follow-up questions were used for clarification, but not to probe beyond the depth of explanation initially offered. In the later interviews this procedure was sometimes relaxed. Clearly the greater flexibility admitted, the less directly comparable the findings from different students, and the further the student responses move from being their spontaneous explanations of phenomena.

However, a direct application of the schedule may sometimes elicit one of a number of available 'explanatory stories' (Millar \& Osborne, 1998), without revealing the availability of alternatives that could be relevant to the level of conceptual integration. That is, the protocol does not allow the researcher to distinguish between a student failing to make a link with relevant and available concepts, and a student failing to report a recognised connection when an available alternative explanation is selected in the interview. Had Benjamin not been asked about a stretching elastic band (not 
part of the pre-planned schedule, see the Appendix) it would not have been revealed that in some contexts he would explain elastic properties in terms of minimising energy rather than forces.

In terms of the case study reported here, it would have been useful to follow-up Alice's 'explanation' of the burning of magnesium, as clearly her response in terms of oxidation did not exclude the possibility of her also being able to demonstrate further thinking about this phenomenon in terms of energy, particles and forces. In the interview she did not spontaneously go on to discuss oxidation in terms that could be linked with her later answers about other reactions and ionisation, but a suitable follow-up question may have readily facilitated this.

As another example, one limitation of the current schedule is that it does not directly test whether students use fully Coulombic models of interactions of charges in some of the contexts. (So Alice used the alternative 'sharing out of nuclear force' idea in the context of the atom but it is not clear this was actually inconsistent with her way of thinking about how "chlorine which is negatively charged [and] sodium which is positively charged" were "attracted to each other".)

The extensive use of follow-up questions can give richer data that may increase the confidence in interpretations that derive from the analysis (especially where manifold conceptions for the same concept area may be held, Taber, 2000a), but limits the number of foci that may be included in an interview, as well as making it more problematic to compare between informants when that is a priority for research. Such methodological decisions often involve careful balancing of antagonistic considerations (Pope \& Denicolo, 1986; Taber, 2002b), again suggesting that the research programme benefits from the accumulation of evidence derived from studies applying complementary research approaches.

There are clearly ways the existing interview schedule could be developed. For example, it would be possible to include phenomena from biological sciences to see if students suggest appropriate links with chemistry and physics topics. Repeated use of the schedule in longitudinal studies could offer insights into the extent of progression in conceptual integration over time. 
Another important direction would be to explore students' own views about conceptual integration. One of the reasons given for the important of this topic is the aim of teaching students about the nature of science. A scientific world-view encompasses an espistemological stance that nature has underlying regularities, that a common set of fundamental laws and principles underpin the natural world. This is not to take a reductionist view that ignores the emergence of new phenomena in complex systems: but rather an expectation that, for example, the principle of conservation of energy will apply in emergent chemical and biological systems regardless of their complexity. The present study looks at the degree of conceptual linkage, but does not tell us whether Alice studies science expecting to find such linkages, and recognises them as a feature of scientific knowledge itself. Such an awareness could both prime students to identify relevant linkages they might otherwise miss, and help them structure their studies when reviewing their class notes and undertaking supportive reading.

\section{Conclusion}

It has been argued here that conceptual integration is an important focus for research both in view of the nature of learning, and the desirability of teaching about the preference for coherence in scientific knowledge systems. Previous research had suggested that even quite advanced and relatively successful learners (Advanced level students in the UK) may struggle to integrate their scientific learning, especially across disciplinary boundaries. In particular, basic physical principles were often ignored by students when learning aspects of chemistry that this physics underpinned.

As one approach to exploring conceptual integration, the common technique of interviewing students about their ideas in science has been adapted for use with a broad-based interview schedule. The schedule asks students for their explanations of a range of phenomena. The schedule was used successfully to interview a small sample of 17-year old college students studying physics and chemistry at advanced level.

One case account is reported here as an example to illustrate the range of information about student thinking that can be elicited in a single interview using this approach. 
Exploring conceptual integration in student thinking: evidence from a case study

Aspects of the case account have been discussed in more detail to highlight some of the diagnostic value of such an interview. In particular the approach offers insight into the extent to which Alice is integrating key ideas in her learning of physics and chemistry. A single interview can only offer a limited window into a students' thinking, so that assumptions about how some of her ideas may be in transition, must remain just that. However, it has been possible to illustrate the potential of the general approach to identifying aspects of conceptual integration.

The methodology used here is only a slight variation on common approaches in science education research. However, I am not aware of this type of broad-based interview being reported before. It is only possible to speculate whether this is because the technique may have seemed problematic (maintaining pace to cover ground, whilst allowing space for full responses; engaging student interest and concentration over a rage of topics); or simply that undertaking this type of investigation has not seemed a priority in the past.

Often in clinical research interviews of this general type, foci are used to engage and stimulate learners (Gilbert, Watts \& Osborne, 1985; White \& Gunstone, 1992) - foci such a line diagrams (interviews-about-instances) or simple demonstrations (interviews-about-events). In the present research simple verbal questions were considered (and found) to be sufficient to stimulate informative responses.

The experience of interviewing Alice and the other students from her college suggests that interviews of this nature are quite feasible and relatively unproblematic to carry out - at least with the caveat that this applies to informants drawn from a population of students who are relatively mature, motivated and successful (and so confident in their abilities). The extent to which the approach could be applied with much younger students, for example, is currently an open question.

Having established the feasibility of the general approach, further stages of the research are suggested. These include comparisons between students to look for general patterns; looking at progression in conceptual understanding by sequences of interviews; and developing the schedule. 
The research programme into learning in science has provided the science education community with a vast amount of material about learners' ideas in thinking, especially in relation to 'snapshots' of how students tend to think about key topics at various ages. The research programme now encompasses more challenging areas of research (Taber, 2006b), and 'conceptual integration' would seem to be one important area where we currently have a very limited research-base. The present paper has reported one approach to exploring the level of integration in students' scientific thinking, offering the account of Alice's thinking across a range of chemistry and physics topics as an example of what such research can uncover. Just as earlier research into student ideas about individual scientific concepts offered insights into effective ways of teaching those topics, it is to be hoped that this new strand of research may ultimately inform teachers on how best to facilitate appropriate conceptual integration among their students.

Acknowledgement: thanks are due to the students, and especially 'Alice', for sharing their thinking, and to Dr. Stephen Martin for assistance in setting up the interviews. 
Exploring conceptual integration in student thinking: evidence from a case study

\section{References}

Ausubel, D. P. (2000) The Acquisition and Retention of Knowledge: a cognitive view, Dordrecht: Kluwer Academic Publishers.

Bell, B. (1995) Interviewing: a technique for assessing science knowledge, in S. M. Glynn, \& R. Duit, (Eds) Learning Science in the Schools: Research Reforming Practice, Mahwah, N.J.: Lawrence Erlbaum Associates, pp.347-364.

DfES (2002) Framework for teaching science: years 7, 8 and 9, Key Stage 3 National Strategy, Department for Education and Skills.

diSessa, A. A. \& Sherin, B. L. (1998) What changes in conceptual change? International Journal of Science Education, 20 (10), pp.1155-1191.

Driver, R., Squires, A., Rushworth, P. \& Wood-Robinson, V. (1994) Making Sense of Secondary Science: research into children's ideas, London: Routledge.

Duit, R. (2006) Bibliography - Students' and Teachers' Conceptions and Science Education, available from http://www.ipn.uni-kiel.de/aktuell/stcse/stcse.html. (accessed 27/04/2006)

Duit, R., Roth, W-M., Komorek, M. \& Wilbers, J. (1998) Conceptual change cum discourse analysis to understand cognition in a unit on chaotic systems: towards an integrative perspective on learning in science, International Journal of Science Education, 20 (9), pp.1059-1073.

Dunbar, R. (1995) The Trouble with Science, London: Faber \& Faber.

Duschl, R. (2000), Making the nature of science explicit, in R. Millar, J. Leach \& J. Osborne (Eds) (2000) Improving Science Education: the contribution of research, Buckingham: Open University Press, pp.187-206.

Engel Clough, E. \& Driver, R (1991) A study of consistency in the use of studnets' conceptual frameworks across different task contexts, in P. Light, S. Sheldon \& M. Woodhead (Eds), Learning to Think, London: Routledge, pp.261-291.

Glaser, B. G. \& Holton, J. (2004) Remodeling Grounded Theory, Forum: Qualitative Social Research, 5(2), Article 4, Available at: http://www.qualitativeresearch.net/fqstexte/2-04/2-04glaser-e.htm

Gilbert, J. K. \& Watts, D. M. (1983) Concepts, misconceptions and alternative conceptions: changing perspectives in science education, Studies in Science Education, 10, pp.61-98.

Gilbert, J. K., Watts, D. M. \& Osborne, R. J. (1985) Eliciting student views using an interview-about-instances technique, in L. H. T. West \& A. L. Pines (Eds), Cognitive Structure and Conceptual Change, London: Academic Press, pp.1127.

Gomm, R., Hammersley, M. \& Foster, P. (2000) Case Study Method, London: Sage.

Goodman, N. (1968) Safety, strength, simplicity, in P. H. Nidditch (Ed.) The Philosophy of Science, London: Oxford University Press, pp.121-123. 
Grevatt, A., Gilbert, J. K. \& Newberry, M. (2007) Challenging able science learners through models and modeling, in Taber, K. S., Science Education for Gifted Learners, Routledge, pp.85-99.

Hammer, David (2004) The variability of student reasoning, Lecture 3: Manifold cognitive resources, Proceedings of the Enrico Fermi Summer School in Physics, Course CLVI, Italian Physical Society.

Harrison, A. G. \& Treagust, D. F. (1996) Secondary students' mental models of atoms and molecules: implications for teaching chemistry, Science Education, 80 (5), pp.509-534.

Harrison, A. G. \& Treagust, D. F. (2000) Learning about atoms, molecules, and chemical bonds: a case study of multiple-model use in grade 11 chemistry, Science Education, 84, pp.352-381.

Harrison, A. G. \& Treagust, D. F. (2002) The particulate nature of matter: challenges in understanding the submicroscopic world, in J. K. Gilbert, O. de Jong, R. Justi, D. F. Treagust \& J. H. van Driel (Eds) Chemical Education: Towards Research-based Practice, Dordecht: Kluwer Academic Publishers, pp.189-212.

Hewson, M. G. A'B. (1985) The role of intellectual environment in the origins of conceptions: an exploratory study, Chapter 10 in West, Leo H. T., and Pines, A. Leon (eds.), Cognitive Structure and Conceptual Change, London: Academic Press Inc., pp.153-161.

Justi, R. \& Gilbert, J. (2000) History and philosophy of science through models: some challenges in the case of 'the atom', International Journal of Science Education, 22 (9), pp.993-1009.

Kuhn, T. S. (1996) The Structure of Scientific Revolutions (3rd edition), Chicago: University of Chicago.

Lakatos, I. (1970) Falsification and the methodology of scientific research programmes, in I. Lakatos \& A. Musgrove (Eds) Criticism and the Growth of Knowledge, Cambridge: Cambridge University Press, pp.91-196.

Lakatos, I. (1978) The Methodology of Scientific Research Programmes, Cambridge: Cambridge University Press, 1978.

Limerick, B., Burgess-Limerick, T. \& Grace, M. (1996) The politics of interviewing: power relations and accepting the gift, International Journal of Qualitative Studies in Education, 9 (4), pp.449-460.

Lijnse, P. L., Licht, P, de Vos, W. \& Waarlo, A. J. (1990) Relating Macroscopic Phenomena to Microscopic Particles: a central problem in secondary science education, Utrecht: Centre for Science and Mathematics Education, University of Utrecht: CD-B Press.

Losee, J. (1993) A Historical Introduction to the Philosophy of Science (3rd Edition), Oxford: Oxford University Press.

McCloskey, M., Carmazza, A. \& Green B. (1980) Curvilinear motion in the absence of external forces: naïve beliefs about the motion of objects, Science, 210, pp.1139-1141. 
Exploring conceptual integration in student thinking: evidence from a case study

Meichtry, Y. (1998) Elementary science teaching methods: developing and measuring student views about the nature of science, in W. F. McComas (Ed.) The Nature of Science in Science Education: Rationales and Strategies, Dordrecht: Kluwer, pp.231-241.

Millar, R. and Osborne, J. (1998) Beyond 2000: Science education for the future, London: King's College.

National Research Council (NRC) (2002) Scientific Research in Education, Committee on Scientific principles for educational research, Washington D.C.: National Academies Press.

Osborne, J. (2002) Learning and teaching about the nature of science, in Amos, S. \& Boohan, R. (eds.) Teaching Science in Secondary Schools: Perspectives on practice, London: RoutledgeFalmer, pp.227-237

Osborne, R. J. \& Wittrock, M. C. (1983) Learning Science: a generative process, Science Education, 67 (4), pp.489-508.

Osborne, R. \& Wittrock, M. (1985) The generative learning model and its implications for science education, Studies in Science Education, 12, pp.59-87.

Petri, J. \& Niedderer, H. (1998) A learning pathway in high-school level quantum atomic physics, International Journal of Science Education, 20 (9), pp.10751088.

Petruccioli, S. (1993) Atoms, Metaphors and Paradoxes: Neils Bohr and the construction of a new physics, Cambridge: Cambridge University Press.

Phillips, D. C. (1987) Philosophy, Science and Social Enquiry: contemporary methodological controversies in social science and related applied fields of research, Oxford: Pergamon Press.

Pope, M. \& Denicolo, P. (1986) Intuitive theories - a researcher's dilemma: some practical methodological implications, British Educational Research Journal, 12 (2), pp.153-166.

Pope, M. \& Gilbert, J. (1983) Personal experience and the construction of knowledge in science, Science Education, 67 (2), pp.193-203.

Popper, K. R. (1982) Quantum Theory and the Schism in Physics, London: Routledge.

Simons, H. (1980) Towards a Science of the Singular: Essays about Case Study in Educational Research and Evaluation, Norwich: Centre for Applied research in Education, UEA.

Smith, J. P, diSessa, A. A. \& Roschelle, J. (1993) Misconceptions reconceived: a constructivist analysis of knowledge in transition, The Journal of the Learning Sciences 3 (2), pp.115-163.

Taber, K. S. (1995) Development of Student Understanding: A Case Study of Stability and Lability in Cognitive Structure, Research in Science \& Technological Education, 13 (1), pp.87-97.

Taber, K. S. (1997) Student understanding of ionic bonding: molecular versus electrostatic thinking?, School Science Review, 78 (285), pp.85-95. 
Taber, K. S. (1998) An alternative conceptual framework from chemistry education, International Journal of Science Education, 20 (5), pp.597-608.

Taber, K. S. (1998) The sharing-out of nuclear attraction: or I can't think about Physics in Chemistry, International Journal of Science Education, 20 (8), pp.1001-1014.

Taber, K. S. (2000a) Multiple frameworks?: Evidence of manifold conceptions in individual cognitive structure, International Journal of Science Education, 22 (4), pp.399-417.

Taber, K. S. (2000b) Case studies and generalisability - grounded theory and research in science education, International Journal of Science Education, 22 (5), pp.469-487.

Taber, K. S. (2001a) Shifting sands: a case study of conceptual development as competition between alternative conceptions, International Journal of Science Education, 23 (7), 731-753.

Taber, K. S. (2001b) Building the structural concepts of chemistry: some considerations from educational research, Chemistry Education: Research and Practice in Europe, 2 (2), pp.123-158.

Taber, K. S. (2002a) Chemical misconceptions - prevention, diagnosis and cure: Volume 1: theoretical background, London: Royal Society of Chemistry

Taber, K. S. (2002) "Intense, but it's all worth it in the end": the colearner's experience of the research process, British Educational Research Journal, 28 (3), 435-457.

Taber, K. S. (2003a) Lost without trace or not brought to mind? - a case study of remembering and forgetting of college science, Chemistry Education: Research and Practice, 4 (3), pp.249-277.

Taber, K. S. (2003b) Understanding ionisation energy: physical, chemical and alternative conceptions, Chemistry Education: Research and Practice, 4 (2), pp.149-169.

Taber, K. S. (2004) Learning quanta: barriers to stimulating transitions in student understanding of orbital ideas, Science Education, 89 (1), pp.94-116.

Taber, K. S. (2005a) Conceptual integration and science learners - do we expect too much?, Invited seminar paper presented at the Centre for Studies in Science and Mathematics Education, University of Leeds, February 2005. The text is in the Education-line internet document collection at: http://www.leeds.ac.uk/educol/documents/00003875.htm

Taber, K. S. (2005) Weak foundations undermine teaching 'scaffolding', Physics Education, 40 (2), pp.115-116.

Taber, K. S. (2006) Towards a Curricular Model of the Nature of Science, Science \& Education, (published on-line first)

Taber, K. S. (2006) Beyond Constructivism: the Progressive Research Programme into Learning Science, Studies in Science Education, 42, pp.125-184. 
Taber, K. S. (2007) Classroom-based Research and Evidence-based Practice: A Guide for Teachers, SAGE Publications.

Tomlinson, P. (1989) Having it both ways: hierarchical focusing as research interview method, British Educational Research Journal, 15 (2), pp.155-176.

Thagard, P. (1992) Conceptual Revolutions, Oxford: Princeton University Press.

Wandersee, J. H. (1990) Concept mapping and the cartography of cognition, Journal of Research in Science Education, 27 (10), pp.923-936.

Watts, D. M. and Zylbersztajn, A. (1981) A survey of some children's ideas about force, Physics Education, 16 (6), pp.360-365.

White, R. T. (1985) Interview protocols and dimensions of cognitive structure, in L. H. T. West \& A. L. Pines (Eds), Cognitive Structure and Conceptual Change, London: Academic Press, pp.51-59.

White, R. \& Gunstone, R. (1992) Probing Understanding, London, The Falmer Press.

Yin, R. K. (2003) Case Study Research: Design and methods (3rd Edition), Thousand Oaks, California: Sage. 


\section{Appendix:}

I'm going to ask you some questions about a variety of phenomena, and I would like you to try and explain them for me:

Could you tell me why you think apples fall to the ground?

Why do you think that apples do not always fall from trees? (What do you think stops them falling?)

Why does a suspended spring stretch when a mass is attached to it?

Why does the spring recoil when the mass is removed?

Why does the lamp in a torch glow when the torch is switched on?

Why do you think current passes through a conductor when it is connected to a source of e.m.f. [power supply] such as a battery?

Why do you think the planets orbit the sun?

Why do you think the moon doesn't move off into space?

Have you seen the party trick where a balloon is rubbed on a jumper/sweater, and then stuck to a wall? (If so) why does the balloon stay attached to the wall?

What happens to a parachutist when she jumps from a plane? (Why do you think she accelerates?) (Why do you think she reaches a constant speed/terminal velocity?)

Have you seen the demonstration where someone holds the dome of a Van der Graaf generator, and their hair stands on end? (Why do you think their hair does that?)

Have you ever seen magnesium burning in air? (You may remember the bright white light as it burns.)

Why do you think magnesium burns in air?

Why do you think chemical reactions occur?

Do you know what the product of the reaction between hydrogen and fluorine is?

Why do you think hydrogen reacts with fluorine to give hydrogen fluoride?

Do you know what the product of the reaction between sodium and chlorine is?

Why do you think sodium reacts with chlorine to give sodium chloride?

Why do you think crystals of sodium chloride don't fall apart? (What do you think holds crystals of sodium chloride together?)

Why do you think chlorine molecules don't fall apart? (What do you think holds chlorine molecules together?)

Why do you think iron crystals don't fall apart? (What do you think holds iron crystals together?)

Why do you think sulfur crystals don't fall apart? (What do you think holds sulfur crystals together?)

Why do you think that ice crystals don't fall apart? (What do you think holds ice crystals together?)

Why do you think ice melts when it is heated?

Why do you think sodium chloride dissolves in water?

What do you think holds [individual] atoms together?

Do you know what the composition [make-up] of an atom of sodium would be? (Can you tell me about the structure [arrangement of parts] of the sodium atom?)

Do you think that a single sodium atom could fall apart? (Could the outer electron fall out of the atom?) (Why?/Why not?) 
Exploring conceptual integration in student thinking: evidence from a case study

Do you think it is possible for a scientist to remove an electron from the sodium atom? (If so: How, do you think?) (If not: Why not?) (What do you think would be left if an electron could be removed from an atom of sodium?)

Do you think it is possible to remove a second electron, i.e. to remove an electron from the sodium ion? (What would be left if the second electron was removed?)

Do you think it is easier/equally difficult/harder to remove the second electron? (Why?)

Do you think it is possible to remove a third electron, i.e. to remove an electron from the sodium ion?

Do you think it is easier/equally difficult/harder to remove the third electron? (Why?)

Do you think that the nucleus of the sodium atom could fall could apart? (Why/why not?)

What do you think holds the protons together in atomic nuclei?

Why do you think some atomic nuclei are unstable? 


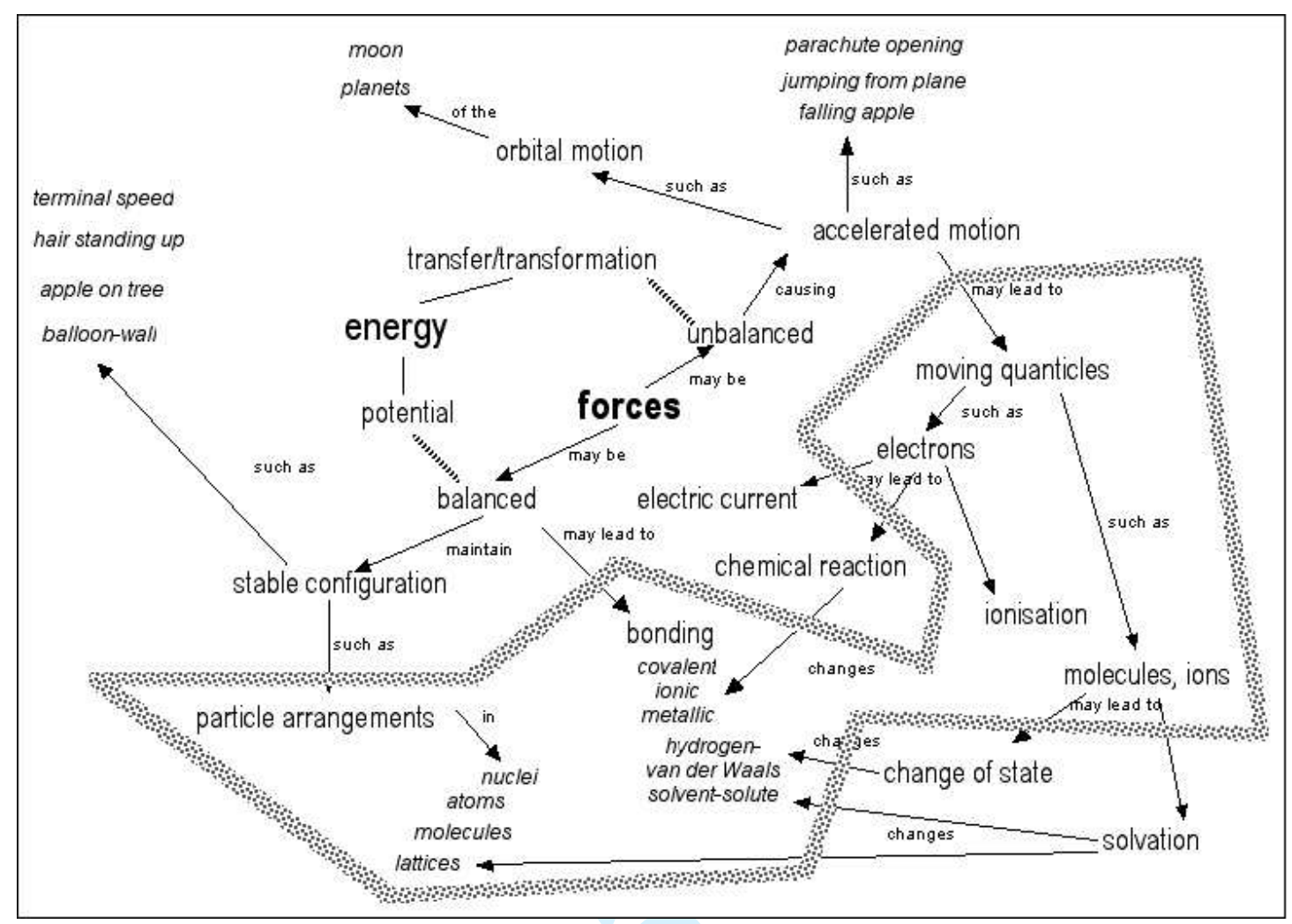

Figure 1: A concept map illustrating themes linking the interview items 
Exploring conceptual integration in student thinking: evidence from a case study

\begin{tabular}{|c|c|c|c|c|}
\hline Assumed name & 'Alice' & 'Ben' & 'Charles' & 'Dorothy' \\
\hline Gender & female & male & male & female \\
\hline $\begin{array}{l}\text { Subjects being } \\
\text { studied }\end{array}$ & $\begin{array}{l}\text { chemistry } \\
\text { physics } \\
\text { biology } \\
\text { German }\end{array}$ & $\begin{array}{c}\text { chemistry } \\
\text { physics } \\
\text { mathematics } \\
\text { English }\end{array}$ & $\begin{array}{l}\text { chemistry } \\
\text { physics } \\
\text { mathematics } \\
\text { German }\end{array}$ & $\begin{array}{c}\text { chemistry } \\
\text { physics } \\
\text { biology } \\
\text { mathematics } \\
\text { Latin }\end{array}$ \\
\hline $\begin{array}{l}\text { intended } \\
\text { University } \\
\text { course }\end{array}$ & $\begin{array}{l}\text { veterinary } \\
\text { science }\end{array}$ & mathematics & $\begin{array}{c}\text { engineering or } \\
\text { chemistry }\end{array}$ & medicine \\
\hline $\begin{array}{l}\text { approximate } \\
\text { interview } \\
\text { duration } \\
\text { /minutes }\end{array}$ & 62 & 50 & 44 & 95 \\
\hline
\end{tabular}

Table 1: The student volunteers interviewed using the schedule 